\title{
LA TERCERA CONFERENCIA DE LAS NACIONES UNIDAS SOBRE EL ESPACIO ULTRATERRESTRE Y EL DERECHO
}

\author{
José Humberto CASTRO VILlalobOs*
}

\begin{abstract}
RESUMEN: Con motivo de la celebración de la UNISPACE III (Tercera Conferencia de las Naciones Unidas sobre la Exploración y Utilización del Espacio Ultraterrestre con Fines Pacíficos) el 19 de julio de 1999, el autor analiza los logros que se han dado en el derecho espacial, desde 1962, año en que se celebró la primera Conferencia hasta nuestros días, haciendo hincapié en aspectos como la teledetección, los desechos espaciales y la protección de los derechos de propiedad intelectual, temas en donde se requiere de la cooperación internacional, para crear mecanismos apropiados que vayan a la par con los rápidos avances tecnológicos.
\end{abstract}

\begin{abstract}
Due to the celebration of UNISPACE III (United Nations Third Conference on Exploitation and Utilisation of Ultraterrestrial Space with Peaceful Means) on July 19, 1999, the author analyses the achievements on Space Law since 1962, when the first conference took place, until the present day, putting emphasis on: teledetection, space waste and the protection to intellectual property rights, matters which require International co-operation to create suitable mechanisms that go along with technological development.
\end{abstract}

* Abogado. Director de Derecho Internacional, Consultoría Jurídica, SRE. Miembro del International Institute of Space Law (IISL). 
SUMARIO: I. Introducción. II. Aspectos generales. III. Codificación del derecho internacional por la Organización de las Naciones Unidas. IV. Régimen jurídico del espacio ultraterrestre. V. Tareas futuras. VI. Conclusiones. VII. Bibliografía.

\section{INTRODUCCIÓN}

La Tercera Conferencia de las Naciones Unidas sobre la Exploración y Utilización del Espacio Ultraterrestre con Fines Pacíficos (UNISPACE III) se realizó en la Oficina de las Naciones Unidas en Viena, Austria, del 19 al 30 de julio de 1999.

Los objetivos principales de UNISPACE III fueron:

a) Promover medios eficaces para utilizar la tecnología espacial para prestar asistencia en la solución de problemas de importancia regional o mundial;

b) Reforzar las capacidades de los Estados, en particular los países en desarrollo, para utilizar las aplicaciones de las investigaciones espaciales en el desarrollo económico y cultural;

c) Proporcionar a los países en desarrollo oportunidades para que definan sus necesidades en materia de aplicaciones espaciales con fines de desarrollo;

d) Considerar formas en que los Estados podrían acelerar la utilización de las aplicaciones espaciales para promover el desarrollo sostenible;

e) Estudiar diversas cuestiones relacionadas con la formación, la capacitación y la asistencia técnica en ciencia y tecnologías espaciales;

f) Ofrecer un foro útil para realizar una evaluación crítica de las actividades espaciales y aumentar la sensibilidad de la población en general respecto de los beneficios de la tecnología espacial, y

g) Fortalecer la cooperación internacional en el desarrollo y la utilización de la tecnología y las aplicaciones espaciales ${ }^{1}$

Como es conocido, uno de los aspectos más exitosos en el campo de la cooperación internacional en materia espacial ha sido, desde luego, la conformación a lo largo de las últimas cuatro décadas del derecho inter- 
nacional del espacio, o sea, del marco legal que regula las actividades de los Estados en la exploración y la utilización del espacio ultraterrestre. La celebración de UNISPACE III fue ocasión propicia para la revisión y el examen de la situación actual del derecho del espacio ultraterrestre así como el planteamiento de los nuevos temas que desde el punto de vista legal ingresarán a la agenda legal del espacio. ${ }^{2}$

En los siguientes renglones haremos algunas reflexiones sobre diversos aspectos de derecho espacial que guardan relevancia con las actividades espaciales y cuyo apropiado tratamiento servirá para proporcionar un marco útil para mejorar la cooperación internacional en este campo.

\section{ASPECTOS GENERALES}

En un ensayo ya clásico intitulado La codification du droit international et les problèmes de sa réalisation, ${ }^{3}$ el profesor Roberto Ago había particularmente desarrollado la idea sobre la necesidad de dar un nuevo impulso a la labor de codificación del derecho internacional. En ese sentido, el más ilustre de los internacionalistas italianos desde Anzilotti se refería a la codificación entendida en su sentido más completo, a saber: una operación consistente esencialmente en reemplazar un derecho de naturaleza costumbrista formado de una manera espontánea a lo largo de los siglos por la opinio iuris de los miembros de la comunidad internacional, por un derecho de formación voluntario, consagrado en los textos escritos adoptados por tales miembros o, al menos, por una mayoría altamente representativa. Esta operación debía de referirse, en primer lugar, a los grandes capítulos tradicionales del derecho de gentes, a saber: las materias fundamentales reguladas todavía por un conjunto de reglas no escritas, de origen costumbrista, heredadas esencialmente de épocas pasadas.

Para el juez Ago, una parte esencial y finalmente irreemplazable del derecho es aquella que tiene su origen no en los actos voluntarios de creación y a veces de imposición, pero que se inscribe espontáneamente e idealiza en la conciencia de los miembros de una sociedad humana determinada bajo el impulso de exigencias concretas y reales. Es este derecho cuya existencia se establece no por la vía de la deducción de un texto es-

2 Benko, Marietta and Schrogl, K. U., Space Law at UNISPACE III and beyond, IISL, 1997.

3 Ago, Roberto, Le Recueil d'études de droit international en hommage à Paul Guggenheim, 1968, p. 23. 
crito y de la investigación de la voluntad más o menos real de sus autores, sino por la vía de la inducción y de la abstracción consecutiva, sobre la base de una serie de comportamientos concretos de los miembros del cuerpo social, de manifestaciones externas y globalmente concluyentes de su opinio iuris. ${ }^{4}$

\section{CODIFICACIÓN DEL DERECHO INTERNACIONAL POR LA ORGANIZACIÓN DE LAS NACIONES UNIDAS}

Sustentado en el artículo 13.1 de la Carta de la Organización de las Naciones Unidas, la Organización Universal ha impulsado de manera decidida el proceso del desarrollo progresivo y la codificación del derecho internacional en diferentes campos, lo que ha sido reconocido como uno de sus mayores logros en el acometido de sus propósitos enunciados en la carta. A lo largo de medio siglo de existencia, la Organización ha pasado revista al corpus iuris gentium existente y ha propiciado una verdadera codificación del derecho internacional dotándolo por primera vez en su historia de una alta dosis de universalidad.

Como es natural — escribió Jorge Castañeda - anteriormente la participación de los Estados débiles en la vida internacional fue menos intensa que la de las potencias. Por ello su contribución a la formación de prácticas y, por lo tanto, de normas jurídicas de origen consuetudinario fue mucho más limitada. Ello contrasta con el pasado, precisamente en la época de creación más vigorosa del derecho internacional donde su aportación fue casi nula. ${ }^{5}$

Este proceso ha sido posible debido a diversas razones que todas ellas conjuntadas han permitido un impulso incuestionable del derecho internacional y que han hecho posible que ocupe un lugar importante, aunque aún no preponderante, en las relaciones internacionales de hoy día.

Esto ha sido así porque en las Naciones Unidas se encuentran representados la casi totalidad de los Estados que conforman hoy la comunidad internacional en su conjunto, lo que ha permitido que las normas jurídicas por ella impulsadas recojan los intereses y las aspiraciones de una inmen-

4 Ago, Roberto, Nouvelles réflexions sur la codification du droit international. International Law at Time of Perplexity, Essays in Honour of Shabtai Rosenne, Dordrecht, Nihhoff Publischer, 1989 , p. 2.

5 Castañeda, Jorge, "Naciones unidas y derecho internacional”, Foro Internacional, México, núm. 2, octubre-diciembre de 1960, p. 224. 
sa mayoría de los actores internacionales, en contraposición al pasado mediato, donde el derecho internacional fue sobre todo un derecho creado por las potencias europeas e impuesto a otros continentes, con el argumento bastante convincente de las bayonetas. Muchas de las instituciones internacionales vigentes desde los siglos XV y XVI habían sido concebidas por los Estados europeos en defensa de sus propios y muy particulares intereses. Estas circunstancias han llevado a algunos autores a cuestionar la existencia de un verdadero derecho internacional cuyas características estaban limitadas a regular a un número muy selecto de actores internacionales. Esto ha cambiado de manera radical a partir de 1945, con la existencia de las Naciones Unidas y con el proceso de descolonización seguido, lo que ha permitido que los miembros de la Organización pasen de cincuenta de 185 Estados miembros. Con todo y las limitaciones a que ha tenido que enfrentar, la organización internacional ha desempeñado un papel más que relevante en la conformación del derecho internacional contemporáneo.

En segundo lugar, las técnicas en la elaboración de las reglas internacionales y su negociación han permitido consagrar un derecho internacional más equilibrado y ponderado que recoge, a su vez, los puntos de vista y articule de una manera más apropiada los intereses generales por encima de los intereses particulares, como sucedió frecuentemente antaño. ${ }^{6}$ Papel relevante en estos propósitos ha sido el desempeño tenido por los diferentes órganos especializados de las Naciones Unidas encargados de la codificación y el desarrollo del derecho internacional público y privado en sus diferentes provincias.

Entre dichos órganos es necesario mencionar a la Comisión de Derecho Internacional de las Naciones Unidas (CDI), la Comisión de las Naciones Unidas para el Derecho Mercantil Internacional (CNUDMI), la Comisión sobre la Condición Jurídica y Social de la Mujer, la Comisión de Derechos Humanos, la Comisión de Prevención del Delito y Justicia Penal y la Subcomisión de Asuntos Legales de la Comisión sobre la Utilización del Espacio Ultraterrestre con Fines Pacíficos. Otros órganos que

6 Como afirmó hace más de cuarenta años el notable jurista y diplomático mexicano Luis Padilla Nervo: "la solución no consiste tanto en reprochar a los nuevos países su escasa vocación jurídica, cuanto en facilitarles el acceso a la creación del derecho internacional. En la medida en que se formulen nuevas normas internacionales, no meramente jurídicas porque reflejen una práctica, sino justas, que tomen también en cuenta las aspiraciones de los nuevos Estados, éstos se inclinarán más a someterse voluntariamente a su aplicación", Anuario de la Comisión de Derecho Internacional, vol. I, 1957, p. 166. 
aun cuando no pertenecen al Sistema de las Naciones Unidas guardan una estrecha vinculación con él, son: la Conferencia de La Haya de Derecho Internacional Privado y el Instituto Internacional para la Unificación del Derecho Privado (UNIDROIT).

\section{Codificación del derecho del espacio ultraterrestre}

La codificación del derecho del espacio ultraterrestre ha tenido lugar a través de métodos de comités ad hoc que tienen un carácter permanente, como es el caso del Comité sobre la Utilización del Espacio Ultraterrestre con Fines Pacíficos ${ }^{7}$ y su Subcomisión Jurídica. Dichos órganos son los encargados por la Asamblea General de preparar proyectos de convenciones y de declaraciones generales sobre estas materias y después sometidos a aquéllas para su aprobación.

El derecho del espacio ultraterrestre formulado por las Naciones Unidas refleja la importancia que la comunidad internacional otorga a la cooperación internacional en la exploración y utilización del espacio ultraterrestre. Hasta ahora, se han redactado cinco tratados y cinco conjuntos de principios jurídicos sobre asuntos relativos a la utilización del espacio ultraterrestre con fines pacíficos gracias a la labor de la Subcomisión de Asuntos Legales de la Comisión del Espacio.

Los cinco tratados son los siguientes: el Tratado sobre los principios que deben regir las actividades de los Estados en la exploración y utilización del espacio ultraterrestre, incluso la Luna y otros cuerpos celestes, que entró en vigor el 10 de octubre de 1967; el Acuerdo sobre el salvamento y la devolución de astronautas y la restitución de objetos lanzados al espacio ultraterrestre, que entró en vigor el 3 de diciembre de 1968; el Convenio sobre la Responsabilidad internacional por daños causados por objetos espaciales, en vigor desde el 1 de septiembre de 1972; el Convenio sobre el registro de objetos lanzados al espacio ultraterrestre, que entró en vigor el 15 de septiembre de 1976; y el Acuerdo que debe regir las actividades de los Estados en la Luna y otros cuerpos celestes, que rige desde el 11 de julio de 1984.

Las cinco declaraciones de principios jurídicos son: la Declaración de los principios jurídicos que deben regir las actividades de los Estados en

7 La Comisión fue establecida por la resolución 1348 (XIII), el 13 de diciembre de 1958, y comprendía en un primer momento a 18 Estados, y posteriormente a 24 Estados. Actualmente está conformada por 54 Estados. 
la exploración y utilización del espacio ultraterrestre, resolución 1962 (XVIII) de la Asamblea General de 13 de diciembre de 1963; los Principios que han de regir la utilización por los Estados de satélites artificiales de la Tierra para las transmisiones internacionales directas de televisión, resolución 37/92 de la Asamblea General, de 10 de diciembre de 1982; los Principios relativos a la teleobservación de la Tierra desde el espacio, resolución 41/65 de la Asamblea General, de 3 de diciembre de 1986; los Principios pertinentes a la utilización de fuentes de energía nuclear en el espacio ultraterrestre, resolución 47/68 de la Asamblea General, de 14 de diciembre de 1992; y la Declaración sobre la cooperación internacional en la exploración y utilización del espacio ultraterrestre en beneficio e interés de todos los Estados, teniendo especialmente en cuenta las necesidades de los países en desarrollo, resolución 51/122 de la Asamblea General, de 13 de diciembre de 1996.

Los principios jurídicos internacionales contenidos en los cinco tratados estipulan, entre otras cosas, que el espacio ultraterrestre pertenece a toda la humanidad y no es susceptible de apropiación por ningún país, y garantizan la libertad de exploración. Prohíben el emplazamiento y la utilización de armas nucleares en el espacio ultraterrestre y prevén la responsabilidad por daños causados por los objetos espaciales y los astronautas, la prevención de interferencias dañinas en las actividades espaciales, la prevención de la contaminación nociva de los cuerpos celestes y los cambios adversos en el medio ambiente de la Tierra, la notificación de las actividades espaciales, el registro de los objetos lanzados al espacio, la investigación científica y la explotación de los recursos naturales, así como la solución de las controversias. Todos los tratados hacen hincapié en la noción de que la jurisdicción sobre el espacio ultraterrestre, las actividades que se realicen en él y cualquier beneficio que pueda derivarse de ello deberán redundar en beneficio de todos los países y de la humanidad, e incluyendo elementos basados en el principio de la promoción de la cooperación internacional en las actividades realizadas en el espacio.

Las cinco declaraciones y conjunto de principios jurídicos aprobados por la Asamblea General de la ONU, por su parte, prevén la aplicación del derecho internacional y la promoción de la cooperación y comprensión internacionales en las actividades espaciales, la difusión y el intercambio de información por conducto de la televisión directa vía satélite y el intercambio de datos e información procedentes de la teleobservación, 
así como normas generales que rigen la utilización segura de las fuentes de energía en el espacio.

El proceso de conformación del derecho del espacio ultraterrestre ha seguido demostrando que es un derecho esencialmente convencional que ha debido conjugar una gran dosis de cooperación política de parte de las potencias espaciales para que haya podido cristalizar. Estas últimas, contrariamente a sus posiciones que han podido adoptar en otros campos de las relaciones internacionales, en el terreno espacial han podido alcanzar acuerdos sobre cuestiones claves. Como prueba de ello puede decirse que hasta 1979 todos los acuerdos y las convenciones adoptadas en el seno de la COPUOS fueron adoptados por consenso. Fue solamente en el caso de los Principios sobre la Teledifusión que la Comisión del Espacio tuvo que recurrir al voto para aprobar el citado instrumento.

Se pueden distinguir varias razones que han permitido el éxito con el que hasta ahora el Comité del Espacio ha podido cumplir su acometido tendiente a formular un régimen jurídico aplicable al espacio ultraterrestre. En primer lugar habría que considerar el interés manifiesto de las potencias espaciales hacia la configuración rápida del derecho del espacio. La ausencia de precedentes ha contribuido de una manera incontestable, ya que al no disponerse de una experiencia bien establecida en el terreno espacial no existían posiciones predeterminadas de los Estados que hubieran influido en la negociación. No existía una doctrina espacial, y los negociadores ponderaban sus posturas, pues sabían que jurídicamente estaban innovando hacia el futuro.

La ausencia de un interés propio por parte de los terceros Estados que no veían las consecuencias inmediatas de las actividades espaciales, así como la imposibilidad para ello de actuar en este terreno vistos los enormes costos económicos y vista la necesidad de contar con una tecnología que se encontraba fuera de sus posibilidades, facilitaron enormemente la tarea codificadora.

Como prueba de lo mucho que ha avanzado el desarrollo del espacio ultraterrestre, cuando el Comité comenzó a considerar materias que por sus propias características afectaban de una manera directa a un gran número de Estados, como fue el caso de la teledetección o de la televisión directa, las posiciones se politizaron y en consecuencia cada vez fue más difícil arribar a normas jurídicas por consenso, por lo que fue necesario recurrir al voto para transigir las posiciones. 
Otro elemento decisivo en el éxito del proceso de codificación del derecho del espacio fue el alto nivel científico y profesional tanto de los miembros del secretariado del Comité del Espacio y de la Subcomisión Jurídica, como de los representantes de los Estados que participaron en las primeras negociaciones. Sobre el particular, la notable contribución del profesor Manfred Lachs, bajo cuya dirección la Subcomisión de Asuntos Jurídicos sentó las primeras bases del derecho espacial durante los primeros quince años y su trabajo pionero en compañía de otros brillantes juristas fue coronado con la adopción en 1967 del Tratado del Espacio, denominado con razón las Doce Tablas del Espacio Ultraterrestre. ${ }^{8}$

\section{RÉGIMEN JURÍDICO DEL ESPACIO ULTRATERRESTRE}

Se ha afirmado que "el espacio ultraterrestre, incluso la Luna y otros cuerpos celestes, no pueden someterse a una apropiación nacional reclamando la soberanía, por medio del uso o de la ocupación, ni por ningún otro medio". ${ }^{9}$ De esa manera se impide a los Estados extenderse y ejercer en esa dimensión los derechos que constituyen los atributos de la soberanía territorial.

La norma en cuestión menciona específicamente dos términos: uso y ocupación. El primero no pertenece a los derechos tradicionales ejercidos sobre el territorio reconocidos por el derecho internacional, mientras que el segundo fue frecuentemente invocada por los Estados en épocas pasadas.

Sin embargo, dice Lachs, no hay necesidad de entrar en un análisis teórico más profundo de este tema; ni el uso ni la ocupación pueden constituir títulos legales para justificar la extensión de los derechos de soberanía de los Estados sobre el espacio ultraterrestre, la Luna y otros cuerpos celestes. La exclusión de estas reclamaciones se complementa con la de apropiación por cualquier otro medio. ¿Que otros medios podrían ser? Se puede citar el descubrimiento. No obstante, desde hace mucho tiempo el derecho internacional ha considerado a éste un derecho incoado que requiere complementarse con otro más válido. ${ }^{10}$

Puede también citarse la vecindad. La ocupación de una pequeña porción de territorio en el pasado sirvió para justificar la reclamación de

8 Jiménez de Aréchaga, E., El derecho internacional contemporáneo, Madrid, Tecnos, 1980, p. 264.

9 Artículo II del Tratado del Espacio de 1967.

10 Lachs, Manfred, El derecho del espacio ultraterrestre, México, FCE, 1977, p. 62. 
áreas mayores. Pero desde el conocido arbitraje sobre Las Palmas se reconoció de forma categórica que: el derecho de vecindad, considerado como base de la soberanía territorial, no tiene fundamento en el derecho internacional.

Por consiguiente, sin importar el criterio que se adopte, se considera que estas reclamaciones no tienen fundamento legal. Por ello, la no apropiación es la norma. Difícilmente podría afirmarse que, como reflejo de la voluntad de los Estados que la incorporaron a un tratado, ésta es una norma de carácter convencional, con todas sus consecuencias, porque las reglas básicas que definen el status del espacio ultraterrestre son normas de derecho internacional general. Esta conclusión se basa en el conjunto total de los derechos y los deberes de los Estados, convencionales y consuetudinarios. Por lo tanto, es difícil pensar que rompiendo un convenio, un Estado pueda tener libertad de acción o el derecho de extender su soberanía al espacio ultraterrestre. ${ }^{11}$

Por otro lado, "el espacio ultraterrestre, incluso la Luna y otros cuerpos celestes, podrán ser libremente explorados y usados por todos los países sin discriminación de ningún tipo, basándose en la igualdad y de acuerdo con el derecho internacional" ${ }^{12}$ Por consiguiente, reafirmando la libertad de acción de los Estados, el Tratado especifica las condiciones de su uso mediante los tres elementos de la definición: $a$ ) la prohibición de la discriminación; $b$ ) el reconocimiento de la igualdad de todos los Estados, y c) el requisito de que las actividades se realicen de acuerdo con el derecho internacional.

Puede afirmarse que estos tres elementos se complementan; la igualdad no permite la discriminación, ni tampoco el derecho internacional, que también requiere de la igualdad. Sin embargo, debe tomarse en cuenta que actualmente sólo unos cuantos Estados se dedican a la exploración del espacio ultraterrestre. Este hecho no puede ser alterado por el derecho. No obstante, sí puede negarse a sancionar las situaciones que puedan impedir que todos los Estados tengan iguales derechos y beneficios en el futuro.

Aunque muy pocos países usan hoy el espacio ultraterrestre, hay varios que realizan experimentos y exploran algunas partes del espacio. Así quedan protegidos sus derechos. Se pone aún más énfasis en esto mediante la disposición legal que afirma que los Estados realizarán todas sus ac-

11 Ibidem, p. 63.

12 Artículo I.2 del Tratado de 1967. 
tividades en el espacio exterior, incluso la Luna y otros cuerpos celestes, con el debido respeto a los intereses de todos los demás Estados. Estos intereses son producto de reconocer los derechos de los otros Estados, y deben traducirse interpretando razonablemente estos derechos. Constituyen los límites de la libertad de acción de los Estados en el espacio ultraterrestre.

La libertad de acceso a todas las áreas de los cuerpos celestes confiere a todos los Estados el derecho de establecer estaciones e instalaciones para realizar experimentos y usar cualquier parte de un cuerpo celeste o su totalidad. La igualdad requiere que este derecho sólo se ejerza en la medida en que sea compatible con el respeto a los derechos y a los intereses propios de los otros Estados. ${ }^{13}$

Dentro del marco de estas consideraciones debe mencionarse especialmente el uso y la exploración eventuales de los recursos y las riquezas naturales. Ni la prioridad en el descubrimiento ni el dominio de la técnica pueden constituir un título para tener derechos exclusivos en este campo. Los países que disponen de medios especiales sin duda pueden reclamar que deben tomarse en cuenta sus esfuerzos que llevaron al descubrimiento y al uso de medios o los recursos. Sin embargo, esto no afecta los principios básicos de que la Luna y los cuerpos celestes no pueden ser objeto de una apropiación nacional, y que la exploración y el uso del espacio ultraterrestre y de los cuerpos celestes se realizará en beneficio e interés de todos los países.

El espacio ultraterrestre y los cuerpos celestes deben considerarse esferas de la actividad de los Estados; como un medio ambiente sujeto a un régimen legal especial, y que goza de una protección particular del derecho internacional. Esto determina la relación entre los Estados y la nueva dimensión. ${ }^{14}$

\section{Tercera Conferencia sobre el Espacio Ultraterrestre}

La Tercera Conferencia de las Naciones Unidas sobre el Espacio Ultraterrestre de 1999 pasó revista al estado actual de la cooperación internacional en la materia y, desde luego, se ocupó del derecho del espacio ultraterrestre haciendo un valioso análisis sobre sus desarrollos más recientes y de las tareas que le esperan a futuro. Como era de esperarse, el

13 Lachs, El derecho, op. cit., nota 10, p. 70.

14 Ibidem, p. 74. 
diagnóstico que realizó UNISPACE III sobre el derecho del espacio constituye una guía inapreciable lo mismo para los especialistas que para el público en general. A continuación se realiza una breve síntesis de las consideraciones que sobre el derecho del espacio realizó el concilio sobre el espacio.

El derecho internacional desarrollado por las Naciones Unidas a través del Comité sobre la Utilización del Espacio Ultraterrestre con Fines Pacíficos y su Subcomité Legal refleja la importancia que la comunidad internacional atribuye a la cooperación internacional en la exploración y la utilización del espacio exterior. Además, cinco tratados y cinco declaraciones sobre principios legales en la materia demuestran cómo las Naciones Unidas han establecido de manera gradual un régimen jurídico que regula las actividades del hombre en el cosmos.

El marco jurídico sobre el espacio dispone que las actividades desarrolladas en dicho ámbito deben efectuarse en provecho de toda la humanidad, y que el espacio exterior incluyendo la Luna y otros cuerpos celestes no están sujetos a ninguna forma de apropiación nacional. Dicho marco normativo consagra también la libertad de exploración del espacio. Se prohíbe el emplazamiento de armas nucleares y cualesquiera otra clase de armas de destrucción en masa y se establece la responsabilidad internacional de los Estados por las actividades nacionales emprendidas en el espacio, así como por los riesgos que puedan derivarse de tales actividades y que lleguen a causar un daño. Igualmente, se contempla un mecanismo para la salvaguarda y el rescate de los astronautas, así como la prevención de actividades que puedan ocasionar daño al medio ambiente de los cuerpos celestes e incluso por efectos perniciosos que puedan repercutir en la Tierra.

Asimismo, se dispone la aplicación del derecho internacional y la promoción de la cooperación internacional, en la realización de las actividades espaciales específicas, como en el caso de la trasmisión y el intercambio de información a través de la televisión directa o de la teleobservación de la Tierra y de sus recursos naturales y de la regulación de la utilización de la energía nuclear desde el espacio.

Por otra parte, la Comisión del Espacio y su Subcomisión Jurídica están considerando de forma concurrente la cuestión de revisar y actualizar los principios relativos al uso de la energía nuclear en el espacio. Igualmente, el tema de la definición y la delimitación del espacio ultraterrestre y el carácter y la utilización de la órbita geoestacionaria, incluyen- 
do las formas de asegurar su uso de manera racional y equitativa por los Estados, sin perjuicio del papel que le corresponda en este campo, a la Unión Internacional de Telecomunicaciones (UIT). Otra cuestión que actualmente se encuentra en la agenda de la Comisión es la revisión del status legal de los cinco instrumentos internacionales que regulan el espacio exterior.

Otras organizaciones intergubernamentales, en particular las del Sistema de las Naciones Unidas, han contribuido a conformar el régimen legal que regula la cooperación internacional en las actividades espaciales. Entre ellas encontramos a la UIT, la Organización Internacional de la Propiedad Intelectual, la Organización Internacional de Meteorología y la Organización Internacional de Energía Atómica. Además, acuerdos bilaterales y multilaterales han venido a coadyuvar al fortalecimiento y a la operación de organizaciones internacionales de carácter regional, como es el caso de la Asociación Espacial Europea, la Organización Internacional de Comunicaciones por Satélite, la Organización Árabe de Comunicaciones por Satélite, la Organización Europea para la Explotación de Satélites Meteorológicos y la Organización Internacional de Comunicaciones por Satélite. Algunos Estados individualmente o asociados a otros Estados han contribuido de manera significativa al desarrollo del corpus del derecho del espacio mediante la adopción de leyes nacionales y de acuerdos que regulan sus actividades en el espacio ultraterrestre y persiguen una mayor cooperación internacional en esta materia.

En años recientes el incremento de las actividades espaciales ha provocado nuevos riesgos en las mismas y creado la necesidad de desarrollar nuevas tecnologías para hacerles frente, como es el caso de los desechos espaciales, el uso de fuentes de energía nuclear en el espacio y la protección de los derechos de propiedad intelectual. Estas materias plantean muchas cuestiones legales que obligan a encontrar soluciones creativas, a través del derecho y la cooperación espacial, que deben mantenerse a la vanguardia de los avances científicos y tecnológicos. Además, las soluciones que exigen estos nuevos desafios deberán tener en cuenta las necesidades de las naciones menos adelantadas.

Las innovaciones en las tecnologías espaciales han conducido a la posibilidad de que en fechas próximas sea realmente posible explotar los recursos naturales del espacio y de los cuerpos celestes. En virtud de la aparente ausencia de consenso sobre los principios jurídicos que se aplican a la Luna y a otros cuerpos celestes, por el bajo número de adherentes 
del acuerdo respectivo, parece necesario multiplicar los esfuerzos tendientes a encontrar fórmulas jurídicas que permitan el acceso a dichos recursos y se garantice su aprovechamiento de manera equitativa para el mayor número de Estados.

Desde la celebración de UNISPACE 1982, el mundo ha sido testigo de un aumento considerable en la comercialización y la privatización de las actividades vinculadas con el espacio exterior. A ello puede añadirse el incremento en el número de actores distintos a los Estados involucrados en dichas actividades, así como a la diversidad de las tareas que comprenden. Telecomunicaciones por satélite, satélites de navegación y de localización, equipo de lanzamiento y de servicios, teledetección, son algunas de las actividades que han experimentado uno de los mayores crecimientos de la industria privada a nivel internacional. Similares actividades como el turismo espacial, la extracción de minerales en asteroides y otros cuerpos celestes y la cada vez más numerosa presencia de la basura espacial requieren de una mayor incidencia de las empresas privadas en el espacio, cuya participación tenderá a incrementarse en un futuro próximo. Estas acciones revisten nuevos riesgos y exigen un tratamiento jurídico imaginativo.

Los Estados miembros de las Naciones Unidas deben iniciar discusiones y buscar soluciones a problemas legales emergentes de trascendencia y, en particular, reconocer la necesidad de considerar la expansión del papel de las empresas privadas cuando dicten nueva legislación internacional sobre el espacio. Las agencias especializadas deben, igualmente, considerar proyectos uniformes y recomendaciones prácticas, así como modelos de sociedades que involucren a capitales públicos y privados en sus respectivos sectores de actividad espacial. El concepto de servicio público y sus variadas manifestaciones debe desarrollarse poniendo una atención particular al interés público global y a las necesidades de las naciones menos adelantadas. El principio de libre comercio debe ser fortalecido. Una cuidadosa atención debe dirigirse a los aspectos de la responsabilidad y la seguridad derivados de la propiedad de vehículos y naves espaciales.

También, los Estados deben considerar el desarrollo efectivo de los mecanismos para la solución de controversias que se produzcan en relación con la comercialización del espacio. Estos mecanismos deberán tomar en cuenta las reglas arbitrales usadas en la práctica internacional para el arreglo de disputas. 
El hecho de que muchos Estados no son partes de varios tratados del espacio ultraterrestre concluidos en el marco de las Naciones Unidas ha venido a minar su autoridad normativa. La Comisión del Espacio y la Subcomisión Jurídica han solicitado la opinión de los Estados sobre los obstáculos que les ha impedido la ratificación de dichos tratados y comenzado a revisar el status de esos instrumentos, con vistas a iniciar una discusión sobre su situación. Este ejercicio ha puesto de manifiesto, además, el hecho de que la adhesión de la práctica de los Estados a las disposiciones de dichos tratados de los que son partes es insatisfactoria, por lo que esta cuestión requiere de una inmediata consideración. Al respecto, parece necesario que los Estados aseguren que su legislación nacional se conforme rigurosamente con los tratados en vigor.

Es indudable, finalmente, que el papel del Comité del Espacio y de la Subcomisión Jurídica como mecanismos adecuados para la elaboración de los principios y normas que regulen las necesidades en el espacio ultraterrestre debe ser fortalecido, con vistas a continuar satisfaciendo los requerimientos del rápido avance de la actividad humana en este campo. ${ }^{15}$

\section{TAREAS FUTURAS}

En años recientes, el aumento de las actividades espaciales ha suscitado nuevos problemas de carácter sumamente técnico, como los desechos espaciales, la utilización de las fuentes de energía nuclear en el espacio y la protección de los derechos de propiedad intelectual. Estos temas plantean muchas cuestiones jurídicas nuevas que requieren soluciones creativas y flexibles en el marco de la cooperación internacional si el derecho internacional del espacio ha de mantenerse a la par de los rápidos adelantos en materia de tecnología y actividades espaciales. ${ }^{16}$

Las innovaciones en la tecnología espacial también dan lugar a actividades orientadas a la explotación de los recursos naturales en el espacio y en los diversos cuerpos celestes, dentro de un margen de viabilidad. Dada la aparente falta de consenso internacional sobre los principios consagrados en el Acuerdo sobre la Luna, como lo demuestra el relativamente escaso número de ratificaciones, es probable que las cuestiones prácti-

15 Para un estudio más detallado sobre estos temas puede consultarse la publicación reciente: Proceedings of the Workshop on Space Law in the Twenty-firs Century, UNISPACE III Technical Forum, July 1999, United Nations, New York, 2000.

16 A/CONF.184/PC/I, p. 64. 
cas relacionadas con la propiedad de esos recursos y el acceso equitativo a ellos tengan que seguirse examinando a fondo en el contexto del derecho internacional. ${ }^{17}$

\section{Comercialización de las actividades espaciales}

Cuando fue elaborado el Tratado del Espacio no resultaba fácil prever en su justa dimensión la magnitud de las posibilidades que ofrecería a las empresas la explotación del espacio exterior con fines comerciales. Como ejemplo de ello baste recordar que el término explotación no aparece una sola vez en el Tratado.

El artículo VI del Tratado de 1967, al disponer la responsabilidad de los Estados por actividades nacionales en el espacio, sin importar la naturaleza de la entidad que las realiza, formula una solución pragmática que puede reducirse a la libertad de acción de la empresa privada bajo la responsabilidad del Estado respectivo. ${ }^{18}$

En consecuencia, al hablar de comercialización de las actividades espaciales encontramos diversos enfoques e interpretaciones. Así, para algunos autores es preferible hablar de una actividad privada comercial por estimarse que la igualdad de derechos reconocida a los Estados en el Tratado del Espacio con respecto a la exploración y utilización del espacio no alcanzaba a las actividades desarrolladas por las empresas privadas en estos ámbitos. Éstas están subordinadas a los Estados respectivos y requieren autorización de los mismos para operar. ${ }^{19}$

Con base en una interpretación amplia del artículo VI citado, otros autores sostienen que internacionalmente, el marco legal no presenta mayores distinciones entre la actividad estatal y la actividad privada. El problema se reduciría en cada caso en identificar cuál sería el sistema constitucional establecido para la incorporación del derecho internacional al derecho interno.

En el momento actual, por ejemplo, todas las actividades de telecomunicaciones efectuadas en el espacio son de naturaleza comercial y están a cargo de entidades gubernamentales o privadas.

17 A/CONF.184/PC/I, p.64 y 65.

18 Maureen, Williams, "Derecho internacional contemporáneo", Anuario de la Comisión Internacional, vol. I, 1957, p. 178.

19 Bourdunov, V. D., Legal Aspects of Private Activities in Outer Space, Proceedings of IISL, Innsbruck, 1986, p. 154. 
La base para sostener la legalidad de las actividades de las empresas privadas en el espacio se encuentran en los artículos I y VI del Tratado del Espacio, que consagra la libertad de acceso, así como la explotación y utilización, y el artículo 11 del Acuerdo de la Luna. Para ciertos autores, ${ }^{20}$ esas disposiciones constituyen incluso normas de carácter consuetudinario y, por lo tanto, válidas independientemente de la existencia del Tratado.

Que el espacio ultraterrestre está siendo utilizado con fines comerciales es algo que está fuera de toda discusión. En consecuencia, es necesario comenzar a identificar los principios legales que normarán esta actividad cada vez más recurrente por parte de las grandes corporaciones internacionales.

\section{Desechos espaciales}

Una buena treintena de años ha sido suficiente para que el espacio entre en nuestra vida cotidiana. Después del primer bip-bip del Sputnik, el 4 de octubre de 1957, cerca de 4 mil satélites han sido lanzados al espacio. Alrededor de 400 están actualmente en explotación. Ellos transmiten de un continente a otro las conversaciones telefónicas, las imágenes de los noticieros de televisión, o los datos de los equipos de cómputo. Salvan la vida de los marineros en peligro o escrutan la atmósfera, el cielo y el sol para los meteorólogos, los astrónomos, los geógrafos y los militares. ${ }^{21}$

Revés de la medalla, esta rápida colonización del espacio próximo de la Tierra no siempre ha sido hecha de manera apropiada. Ciertas zonas de los alrededores espaciales de nuestro globo se encuentran saturadas de desechos de cohetes espaciales y de satélites comenzando a adquirir proporciones alarmantes. Frecuentemente denunciado los últimos años, esta contaminación se ha vuelto cada vez más preocupante, lo que ha obligado a que el tema sea discutido por expertos cada vez con mayor frecuencia. Así, en una pasada reunión convocada por la Agencia Espacial Europea (ESA) efectuada en Darmstadt, Alemania, 250 especialistas reconocieron que se asiste a una verdadera emergencia de sensibilidad ecológica aplicable al espacio.

Más allá de la moda verde, los ingenieros y juristas especializados participantes en la Conferencia de Darmstadt no tenían más que una sola preocupación: cómo evitar la contaminación creciente de las órbitas más

20 Maureen, Williams, Derecho internacional contemporáneo, op. cit., nota 18, pp. 180-181.

21 Martin, Pierre-Marie, Le droit de l'espace, Paris, Presses Universitaires de France, 1991, p. 17. 
frecuentemente utilizadas que amenaza una actividad cuyo interés económico está fuera de toda duda.

En cuarenta misiones efectuadas hasta mayo de 1991, más de cincuenta trazas de colisión han sido encontradas en los fuselajes de las naves espaciales norteamericanas, lo que ha obligado al remplazo de veinticinco de sus componentes. Tres cuartas partes de estos impactos han sido atribuidos a pequeños fragmentos de desechos espaciales. Pero más aún, el Atlantis (el 28 de diciembre de 1991) y el Discovery (el 16 de septiembre de 1991 y el 8 de diciembre de 1992) han tenido que maniobrar para evitar una colisión con desechos espaciales en pleno vuelo.

Según los especialistas, el riesgo es aún débil, pero real: durante los 17 años que pasó en órbita una gran estructura como el telescopio Hubble tuvo un $4 \%$ de riesgo de ser seriamente dañado por un desecho espacial de origen humano. ${ }^{22}$ Una probabilidad suficientemente elevada para todas las agencias espaciales que trabajan actualmente en blindajes espaciales destinados a equipar las futuras estaciones habitadas o a los grandes satélites destinados a permanecer largo tiempo en órbita baja.

Las cifras hablan por ellas mismas. Los radares de la red de vigilancia espacial del ejército de los Estados Unidos rastrean actualmente a 7,000 objetos espaciales de una dimensión superior a 10 centímetros en órbita baja, o sea de 200 a 1,000 kilómetros de altitud. Según las estimaciones de los expertos, el número de fragmentos comprendidos entre $10 \mathrm{y}$ 1 centímetros, difíciles de ver e imposibles de detectar, pero susceptibles de penetrar un blindaje, podrían sobrepasar los 100 mil. ${ }^{23}$

Se encuentra de todo en órbita. Desde los satélites en funcionamiento o abandonados después de siete diez años de buenos y leales servicios. Pero también los pisos superiores de los cohetes que los han puesto en órbita, hasta herramientas perdidas por los astronautas cuando efectuaban sus salidas en el espacio.

Bien que muy raro el aire subsiste en órbitas bajas en cantidades suficientes para cambiar el curso de los desechos espaciales. A 200 kilómetros de altitud, un desecho de dimensión media no permanece más de cuatro días en órbita antes de desplomarse hacia la Tierra. Si no es muy compactado, el frotamiento con las altas capas de la atmósfera lo carboniza mucho antes de que toque el suelo. Pero este fenómeno de tránsito ae-

22 Johnson, Nicolas and Meknight, Darres S., Artificial Space Debris, 2a. ed., Krieger, 1991, p. 67.

23 Tascione, T. F., Space Environmental Hazards: A Guia to Building Better Spacecraft Orbit, 1991, p. 19. 
rodinámico desaparece rápidamente con la altitud. A 600 kilómetros, un objeto puede permanecer treinta años antes de desplomarse. Su duración de vida podría alcanzar veinte mil años a 2,000 kilómetros. En la órbita geoestacionaria, o sea a 36 mil kilómetros, los satélites de telecomunicaciones podrían durar millones de años.

Cierto, es necesario considerar estas estadísticas a la escala espacial. A pesar del peligro que representa la multiplicación de los meteoritos artificiales que alcanzan velocidades cercanas a los 25 mil kilómetros/hora, los espacios orbitales son vastos y amplios.

Pero los expertos creen que esta situación no durará mucho. ${ }^{24}$ Ellos temen la aparición de reacciones en cadena: un desecho grande, como pudiera ser un cohete de lanzamiento o un satélite abandonado, explotan y se convierten en una multitud de fragmentos que a su vez podrían convertirse en otros desechos; este fenómeno podría saturar las órbitas más frecuentadas con una multitud de pequeñas partículas que las haría rápidamente inutilizadas. Cientos de nuevas fragmentaciones de este género han sido ya observadas. La más espectacular fue sin duda la explosión en 1986 del último piso de un cohete Ariane-4, lo que entrañó la formación de 488 desechos observables.

Para intentar escapar a este escenario catastrófico, los organismos y las sociedades de lanzamiento y de explotación de satélites han tomado espontáneamente ciertas medidas preventivas. Los europeos, a la cabeza en este campo, decidieron vaciar el combustible que queda en el tercer piso del cohete Ariane una vez que éste ha dejado a su satélite en órbita. Estas medidas destinadas a evitar una explosión ulterior han sido tomadas desde 1989 para los lanzamientos en órbita baja. Posteriormente, esta medida ha sido extendida a todo tipo de lanzamientos.

Para el lanzamiento del Ariane-5 con dos pisos ya se ha tomado en cuenta el problema de los desechos desde su concepción. La trayectoria del cohete está calculada para que el primer nivel criogénico caiga en el Océano Pacífico en su primera vuelta en órbita. Este objetivo ha sido alcanzado con una pérdida de 700 kilogramos de impulsores de lanzamiento. Para compensar una parte de la capacidad inicial perdida, el volumen de los depósitos del segundo nivel han sido aumentados. Así, este último será como el Ariane-4, un objeto pasivo al concluir su lanzamiento. 
Rusos, norteamericanos, japoneses y chinos han seguido este ejemplo. Los operadores de satélites de telecomunicaciones se han convencido por elevar sus satélites sin vida útil a una órbita cementerio que, estiman los expertos, deberá estar situada al menos a 300 kilómetros. Este proceso ha sido ya efectuado con mayor o menor éxito más de media centena de veces.

Estas primeras medidas de urgencia son susceptibles, en un primer momento, de reducir la contaminación en el espacio. Pero otras acciones, más radicales, serán necesarias para regular el problema. Un comité ad hoc de expertos de la Academia Internacional de Astronáutica estudia diversas posibilidades: reunir los desechos con la ayuda de una suerte de draga espacial, desplazamiento de la basura a una órbita baja donde podrá descender rápidamente, la destrucción de los fragmentos mediante rayos lasser, etcétera. La solución más atractiva consiste en la fabricación de una especie de remolcador automático de propulsión eléctrica, capaz de pulverizar los desechos más grandes. Aunque esta solución exigirá de investigaciones tecnológicas largas y costosas. ${ }^{25}$

Pese a todo, las agencias espaciales de Europa, los Estados Unidos, Rusia y Japón han decidido coordinarse para el establecimiento de una base de datos común sobre los desechos espaciales. Esto, al menos, es prueba de la existencia de consenso.

La NASA trabaja actualmente sobre medidas para prevenir al máximo la creación de más basura espacial. Los representantes de la agencia rusa RKA se han comprometido en ese mismo sentido. El espacio es actualmente objeto de una batalla económica feroz, y tales medidas cuestan caro. El desplazamiento de un satélite geoestacionario a una órbita cementerio alta, por ejemplo, reduce en tres veces menos su vida útil, que es de siete a diez años de vida en promedio. ${ }^{26}$

Actualmente, las Naciones Unidas realizan estudios con vistas a iniciar en los próximos tiempos un ejercicio diplomático destinado a formular principios jurídicos sobre esta materia. Es necesario desplegar mayores esfuerzos tendientes a crear conciencia, sobre todo, entre los operadores privados y entre los pequeños países, de la urgente necesidad de preservar el espacio de la contaminación humana. ${ }^{27}$

25 Exploration Commerciale de l'Espace-Droit Positif, Droit Prospectif, Dijon, CNRS, Université de Bourgogne, núm. 15, 1992.

26 Shahroki, F. et al., Space Comercialization: Satellite Technological, 1990.

27 Benko, Marieta et al., Space Law in the United Nations, Dordreacht, Nijhoff Publishers, 1985 . 
Desde un punto de vista jurídico, se puede constatar que el principio de la libertad de exploración y de utilización del espacio exterior implica el derecho de las misiones espaciales de no ser expuestas al peligro de los desechos espaciales de misiones anteriores. ${ }^{28}$

La cuestión de la contaminación espacial es aún más preocupante en el caso de la órbita geoestacionaria, donde el número de satélites que pueden ser emplazados es relativamente limitado.

La solución a este problema no puede ser otra que una aproximación interdisciplinaria del problema, donde los aspectos legales, representados por un proceso de consultas que pueden hacer menos agudo el problema deben unirse los medios científicos y técnicos para resolverlo totalmente. ${ }^{29}$

\section{CONCLUSIONES}

La rápida expansión de las actividades privadas en relación con el espacio ultraterrestre requiere del examen de varios aspectos del derecho del espacio:

a) Respecto a la aplicación de los servicios espaciales que pueden originar responsabilidad internacional, responsabilidad por riesgo y casos de jurisdicción concurrentes no cubiertas por el derecho espacial;

b) Impacto de la comercialización y privatización de las actividades espaciales sobre los aspectos del servicio público de tales servicios;

c) Cuestiones de propiedad intelectual y transferencia de tecnología que puedan requerir un tratamiento especial de uniformidad global en la práctica.

d) Protección de los derechos de los inversionistas en actividades espaciales que pueden requerir de nuevas aproximaciones legales para hacerlos efectivos;

e) Nacionalidad de las naves espaciales;

f) Protección del medio ambiente, cuando concurran actividades de las entidades privadas en el espacio. ${ }^{30}$

28 Martin, Pierre-Marie, op. cit., nota 21, p. 60.

29 Idem.

30 Informe de la Tercera Conferencia de las Naciones Unidas sobre la Exploración y Utilización del Espacio Ultraterrestre con Fines Pacíficos,Viena, 30 de julio de 1999 A/Conf. 184/6, p. 153. 
Como el uso de las actividades espaciales proliferarán cada día más, se ha constatado que muchos recursos existentes (órbitas, frecuencias, acceso a infrestructuras en el espacio, etcétera) no son ilimitados. Consecuentemente, tales recursos deben ser utilizados de forma racional y equitativa y sujetos a una administración rigurosa, de conformidad con los principios legales internacionales aplicables. El interés público global en este campo debe ser primeramente salvaguardado por las instituciones públicas. Hay una necesidad primordial de contar con una adecuada coordinación en esta área.

El rápido desarrollo de las actividades espaciales requiere la consideración de un gran número de aspectos. El incremento de las actividades espaciales ha venido afectando determinado tipo de acciones vinculadas al derecho económico internacional, lo que ha provocado la desaparición de las fronteras tradicionales entre derecho público y privado y generado mayor confusión entre los estándares y la práctica seguida hasta ahora. En el terreno del medio ambiente, es importante tener mecanismos apropiados para la solución de controversias que den plena vigencia a los principios del derecho del espacio ultraterrestre de una manera flexible pero puntual.

El crecimiento que han experimentado ciertas áreas como las actividades comerciales derivadas de los servicios de teledetección, su complejidad comercial, y sus efectos sobre la cooperación internacional y las aplicaciones científicas e industriales de los servicios, requieren de reglamentaciones apropiadas. Las restricciones nacionales para el acceso de datos tienden a generalizarse. Por ello es necesario preservar el principio de no discriminación en cuanto al acceso a dicha información.

Varios temas recientes han sido influidos por el rápido avance de la ciencia y la tecnología. El derecho del espacio debe tener una sólida fundamentación en hechos científicos y tecnológicos para asegurar su apropiada formulación legal. La interacción entre expertos científicos y legales debe conducir a reforzar la relevancia del derecho en esta materia.

Finalmente, uno de los cambios más significativos en las actividades espaciales es el relativo a la expansión global de los servicios de navegación por satélites que debe contar con un marco legal efectivo.

\section{BIBLIOGRAFÍA}

AGO, Roberto, Le Recueil d'études de droit international en hommage à Paul Guggenheim, 1968. 
—_, Nouvelles réflexions sur la codification du droit international, International Law at Time of Perplexity, Essays in Honour of Shabtai Rosenne, Dordrecht, Nihhoff Publischer, 1989.

BENKO, Marietta and SCHROGL, K. U., Space Law at UNISPACE III and beyond, IISL, 1997.

— et al., Space Law in the United Nations, Dordreacht, Nijhoff Publishers, 1985.

BourdunOV, V. D., Legal Aspects of Private Activities in Outer Space, Proceedings of IISL, Innsbruck, 1986.

CASTAÑEDA, Jorge, "Naciones Unidas y derecho internacional", Foro Internacional, México, núm. 2, octubre-diciembre de 1960.

Exploration Commerciale de l'Espace-Droit Positif, Droit Prospectif, Dijon, CNRS, Université de Bourgogne, núm. 15, 1992.

Informe de la Tercera Conferencia de las Naciones Unidas sobre la Exploración y Utilización del Espacio Ultraterrestre con Fines Pacíficos,Viena, 30 de julio de 1999 A/Conf. 184/6, p. 153.

JIMÉNEZ DE ARÉCHAGA, E., El derecho internacional contemporáneo, Madrid, Tecnos, 1980.

Johnson, Nicolas and MeKnight, Darres S., Artificial Space Debris, $2 \mathrm{a}$. ed., Krieger, 1991.

LACHS, Manfred, El derecho del espacio ultraterrestre, México, FCE, 1977.

MARTIN, Pierre-Marie, Le droit de l'espace, Paris, Presses Universitaires de France, 1991.

MAUREEN, Williams, "Derecho internacional contemporáneo", Anuario de la Comisión de Derecho Internacional, vol. I, 1957.

Proceedings of the Workshop on Space Law in the Twenty-firs Century, UNISPACE III Technical Forum, July 1999, United Nations, Nueva York, 2000.

TAscione, T. F., Space Environmental Hazards: A Guia to Building Better Spacecraft Orbit, 1991. 